\title{
The helium weak silicon star HR 7224
}

\section{Doppler Imaging analysis ^}

\author{
H. Lehmann ${ }^{1}$, A. Tkachenko ${ }^{2}$, L. Fraga ${ }^{1,3}$, V. Tsymbal ${ }^{2,4}$, and D. E. Mkrtichian ${ }^{5,6}$ \\ 1 Thüringer Landessternwarte Tautenburg, 07778 Tautenburg, Germany \\ e-mail: lehm@tls-tautenburg.de \\ 2 Tavrian National University, Dep. Astronomy, Simferopol, Ukraine \\ e-mail: andr309@yandex.ru; vad@simfi.net \\ 3 Departamento de Física UFSC, CP 476, 88040-900 Florianópolis, SC, Brazil \\ ${ }^{4}$ Institut für Astronomie, Unversität Wien, Türkenschanzstr. 17, 1180 Wien, Austria \\ 5 Astrophysical Research Center for the Structure and Evolution of the Cosmos, Sejong University, Seoul 143-747, Korea \\ ${ }^{6}$ Astronomical Observatory, Odessa National University, Shevchenko Park, Odessa 65014, Ukraine \\ e-mail: davidm@sejong.ac.kr
}

Received 23 April 2007 / Accepted 7 June 2007

\section{ABSTRACT}

\begin{abstract}
Aims. We investigate the abundance distributions of silicon and iron on the surface of HR 7224 and check for the presence of magnetic fields. We try further to find an explanation for the observed Balmer line variations, alternative to that by surface temperature gradients given in a previous article.

Methods. Based on time series of more than 570 high-resolution spectra we investigate the abundance distribution of silicon and iron using the Doppler Imaging technique applying two independent program codes and using single and multiple components atmosphere models. We further calculate the combined effect of abundance changes of $\mathrm{Si}, \mathrm{Fe}$, and $\mathrm{He}$ on the $\mathrm{H}_{\beta}$ line profile and compare it with the observations. The mean longitudinal magnetic field strength of HR 7224 is measured spectropolarimetrically.

Results. The Doppler Imaging analysis of HR 7224 shows the presence of large silicon and iron spots on the star's surface, large gradients of the abundances of these elements, and hints to enhanced line strengths near the visible pole of the star. The explanation of the observed Balmer line variations by inhomogeneous abundance distributions alone requires unrealistic large gradients of the helium abundance. Magnetic field measurements gave no definite results, a field strength of the order of $+400 \mathrm{G}$ is likely.

Conclusions. We can exclude the hypothesis that the hydrogen line profile variations of HR 7224 are caused by inhomogeneous abundance distributions of $\mathrm{Fe}, \mathrm{Si}$, and $\mathrm{He}$ alone or by pressure gradients like in the case of Ap stars. Still the observed variations can be described only by the combined effect of abundance and temperature gradients. In this case, the derived Doppler imaging maps will reflect also the temperature gradients and the surface will be cooler in regions of enhanced line strength like at the visible pole. At present state, the physical origin of possible surface temperature gradients remains unknown.
\end{abstract}

Key words. stars: chemically peculiar - stars: variables: general - stars: atmospheres - stars: abundances

\section{Introduction}

HR 7224 (HD 177410, HIP 93187, EE Dra) is a rapidly rotating CP Si star. It was classified by Crawford (1963) and Cowley et al. (1969) as Ap0 star. The photometric variability of HR 7224 is known since a long time (Winzer 1974). The light curve shows a double wave (Adelman 1997) over the rotation period. A more detailed description of the history of HR 7224 observations is given in Lehmann et al. (2006, Paper I).

In Paper I we investigated the star spectroscopically. The work presented in Paper I was stimulated by an article by Adelman (2004) where he supposed a drastic increase of the rotation period from $1 \mathrm{~d}$ to $100 \mathrm{~d}$ and of the photometric amplitude by a factor of six in the period between 1996 and 2003. From our high-resolution spectra taken in 2005 we deduced a

* Based on observations obtained at the 2-m telescope at the Thüringer Landessternwarte Tautenburg and at the 1.8-m telescope at the Bohyunsan Optical Astronomy Observatory. rotation period of $1.12324 \mathrm{~d}$. We found that HR 7224 has enhanced $\mathrm{Si}$ and Fe abundances whereas He is strongly depleted. The double-wave shaped radial velocity (RV) and line profile variations (LPV) indicate the presence of two large spots on opposite sides of the star. An unusual observation was that all metal lines as well as the Balmer lines vary in strength. We compared the spectra from the rotation phases where the star shows maximum line strength with those from the phases of minimum line strength and tried to fit the observed Balmer and metal lines by synthetic spectra calculated from adapted model atmospheres. We derived $\log g=4.18$ and $v \sin i=100 \mathrm{~km} \mathrm{~s}^{-1}$. Results showed, however, that it was not possible to fit the profiles of the Balmer lines in both phases by varying the abundances of single chemical elements. Only if we assume different temperatures for the two phases $-15500 \mathrm{~K}$ for the phase of minimum and $14500 \mathrm{~K}$ for the phase of maximum line strength were we able to fit the observations. From our atmosphere models we could confirm the high photometric amplitude reported by Adelman (2004) for the star in 2003. 
Surface temperature inhomogeneities had been reported for only very few other stars in this spectral range like for the fast rotating Ap star CU Vir (Kroll 1989) and for $\alpha^{2} \mathrm{CVn}$ (Böhm-Vitense \& Van Dyk 1987). Authors found that $\alpha^{2} \mathrm{CVn}$ is cooler by $1000 \mathrm{~K}$ at the magnetic poles than at the equator and explain the observed photometric changes by a non-spherically symmetric oblique rotator model. These results could not be confirmed by other authors, however (Kuschnig et al. 1999; Kochukhov et al. 2002; see also Sect. 6). We have no physically convincing explanation for the existence of the temperature gradients on HR 7224 so far. Although we showed in Paper I that the observed variations are compatible with the assumption that HR 7224 has cool polar caps that are slightly inclined to the rotation axis, there is no direct analogy to the scenario described by Böhm-Vitense \& Van Dyk since the magnetic field is missing. There was only one attempt to measure the magnetic field of HR 7224 by Bohlender et al. (1993) but without any findings. We used the possibility to get additional magnetic field measurements within a short run at the new spectropolarimeter at the BOES spectrograph (see Sect. 2).

The large amplitude of the rotational RV variation of $\pm 6 \mathrm{~km} \mathrm{~s}^{-1}$ makes the star an ideal candidate for Doppler Imaging (DI) analysis. The spectrum of HR 7224 is dominated by the Si II and Fe II lines. We apply the DI technique to HR 7224 to get a more detailed picture of the surface abundance distributions of the corresponding chemical elements. In particular we want to confirm the presence of the two large spots on opposite sides of the star and the possible existence of polar caps as suspected from the RV and equivalent width $(E W)$ analysis in Paper I. We try further to establish an atmospheric model that explains the observed LPV of the Balmer lines in an alternative way without assuming large temperature gradients on the stellar surface.

\section{Observations and data reduction}

Line profile analysis. For Doppler Imaging (DI) and Balmer lines analysis we used 577 high-resolution spectra of HR 7224 obtained with the coudé-echelle-spectrograph at the 2-m telescope at the Thüringer Landessternwarte in 2005 and a few spectra taken with the fiber-fed Bohyunsan Observatory Echelle Spectrograph (BOES, Kim et al. 2002, 2007) at the $1.8-\mathrm{m}$ telescope at the Bohyunsan Optical Astronomy Observatory (BOAO). Tautenburg spectra have a spectral resolution of 63000 and cover a wavelength range from $4760 \AA$ to $7400 \AA$ whereas the BOES spectra have a resolution of 45000 and range from $3600 \AA$ to $10500 \AA$. A detailed description of observations and data reduction can be found in Paper I.

Magnetic field measurement. High resolution $(R=60000)$ circular polarization spectra have been obtained on February 28 in 2007 with the new spectropolarimetric device at the BOES. Each observation consists of a pair of two consecutive exposures at two orthogonal orientations $\left( \pm 45^{\circ}\right)$ of the quarter-wave plate. We obtained two observations (four spectra) of HR 7224 and two observations (four spectra) of the magnetic field standard star $\alpha^{2} \mathrm{CVn}$ for comparison. The reduction of the BOES spectra was done with the BOES automatic image processing package.

\section{Magnetic field measurement}

For the measurement of the mean longitudinal magnetic field $B_{\text {eff }}$ we used 23 metal lines in the $4100-8870 \AA$ range having depths stronger than 0.1 of the continuum level. The magnetic field strength was determined from the displacement of the centers of gravity of the Zeeman-shifted lines measured from the spectra of opposite circular polarization (e.g. Landstreet 1980; Monin 2002). It is

$\Delta \lambda=9.34 \times 10^{-13} \lambda^{2} g_{\mathrm{eff}} B_{\mathrm{eff}}$

where $g_{\text {eff }}$ is the effective Lande factor of the transition and $\lambda$ and $B_{\text {eff }}$ are measured in $\AA$ and in Gauss, respectively. Atomic data were taken from the VALD data base (Piskunov et al. 1995). In case of the $\mathrm{O}, \mathrm{Si}$, and $\mathrm{Mg}$ lines where the Lande factors are missing in the VALD tables $g_{\text {eff }}$ was calculated in LS coupling. For blended lines $B_{\text {eff }}$ was calculated from the intensity weighted sum of the Lande factors of the components. The relative intensity weights of the components of the blends were calculated using the mean chemical compositions and effective temperatures determined in Paper I for HR 7224 and by Cohen (1970) for $\alpha^{2} \mathrm{CVn}$.

The rapid rotation of the $\operatorname{star}\left(v \sin i=100 \mathrm{~km} \mathrm{~s}^{-1}\right)$ and the small number of spectra and measurable lines (due to blending and the low SN of weak lines) do not permit to measure the magnetic field of HR 7224 with high accuracy. From the two observations we obtain $500 \pm 590 \mathrm{G}$ and $350 \pm 680 \mathrm{G}$. The weighted mean longitudinal magnetic field from both measurements is $430 \pm 450 \mathrm{G}$ and corresponds to a rotation phase of $\Phi=$ 0.529 according to the epoch of maximum RV of $2453520.51 \pm$ $1.123248 E$ as derived in Paper I.

For $\alpha^{2} \mathrm{CVn}$ we obtained two measurements of $840 \pm 110 \mathrm{G}$ and $1090 \pm 50 \mathrm{G}$ at the mean epoch HJD 2454160.1133 corresponding to phase $\Phi=0.5096$ of the ephemeris given by Farnsworth (1932). These measurements are in good agreement with the value of $+900 \mathrm{G}$ expected for the iron lines (Fig. 5 in Wade et al. 2002) and confirm the absence of larger systematic instrumental effects. More detailed results on the quality of the new instrument will be based on more extended measurements of the magnetic field of $\alpha^{2} \mathrm{CVn}$ and will be published in a forthcoming paper.

\section{Doppler imaging analysis}

\subsection{Doppler imaging}

If a star rotates rapidly enough so that the shape of its line profiles is dominated by rotational Doppler broadening, a high degree of correlation exists between the position of any distortion within a line profile and the position of the corresponding local deviation from the mean on the stellar surface (Vogt et al. 1987). DI tries to reconstruct the two-dimensional stellar surface structure from a set of one-dimensional images delivered by a time series of spectra. This is possible in principle if the rotation rate and the inclination of the star are within certain limits. The star has to rotate fast enough so that the profile is dominated by Doppler broadening (the number of resolvable elements on the surface increases with increasing $v \sin i$ ) but it must not be rotating so fast so that lines free of blends cannot be found in its spectrum. Both limits depend on spectral type. HR 7224, where $v \sin i$ is in the order of $100 \mathrm{~km} \mathrm{~s}^{-1}$, is close to the upper end of this velocity scale. The second criterion is that the inclination of the rotation axis of the star is not too large. For $i>70^{\circ}$ the northsouth ambiguity as to which hemisphere a surface feature is on is large and results in Doppler images that have mirror-image symmetry about the stellar equator (Vogt et al. 1987).

DI inversion is a so-called ill-posed problem that possesses an infinite number of solutions. To find a unique solution, the 
inverse problem must be regularized (Piskunov \& Kochukhov 2002). For the DI analysis, we used two different programs, MAXENT (Vogt et al. 1987; Hatzes et al. 1989) and INVERS8 (Piskunov \& Rice 1993). The application of MAXENT was our first attempt to get a more detailed picture of the surface structure of HR 7224. It uses the maximum entropy method (MEM) and ends up with a surface map in terms of equivalent widths $(E W \mathrm{~s})$ that shows a minimum correlation between the surface elements (Piskunov et al. 1990). Since the obtained $E W$ distribution cannot be directly translated into an abundance distribution MAXENT is not as well suited for our purposes as the INVERS 8 code and its results are superseded by the results of the more modern code. Nevertheless we present the basic MAXENT results for comparison, mainly to show the influence of the regularization parameter on the results.

INVERS8 uses Tikhonov regularization that delivers results in the sense of the smoothest map in terms of stellar surface abundances that is possible to fit the observations at a certain level. Both programs use pre-calculated spectra of synthetic intrinsic (rotational un-broadened) lines based on atmosphere models (Fig. 1). We used LLmodels by Shulyak et al. (2004) for the computation of atmosphere models and SynthV by Tsymbal (1996) for the computation of synthetic line spectra. Both programs allow to use individual abundance patterns.

MAXENT and INVERS8 were applied to the same data set. To reduce the influence of blends (due to the large $v \sin i$ of HR 7224 almost all lines are blended with Fe II lines) we decided to use only the strongest Si II lines where blends with other metal lines can be neglected. The data set was built by averaging the 577 line profiles of the Si II lines at 5041, 5056, 6347, and $6371 \AA$ into 25 equally spaced rotation phase bins each. For one single spectrum the typical continuum SN ratio is 140 , for one phase binned spectrum we can estimate it is about 5 times higher. Additionally, we investigated the Fe II $5316 \AA$ line with INVERS8.

The number of stellar surface resolution elements that can be handled by the DI programs is limited only by the amount of computing time that we will allow for. The real resolution that can be obtained is given by stellar properties. To first order, the number of resolution elements across the visible disk will be

$N_{\text {res }}=2 \frac{v \sin i}{W}$

where $W$ is the FWHM of the intrinsic (rotationally unbroadened) line profile (Vogt et al. 1987). Figure 1 shows the intrinsic profiles for two of the lines used, Si II $6347 \AA$ and Fe II $5316 \AA$. The intrinsic line width of the strong silicon lines of about $24 \mathrm{~km} \mathrm{~s}^{-1}$ allows for a resolution in the order of only about 8 resolution elements across the visible surface corresponding to a resolution of about $23^{\circ}$ in stellar longitude. In case of the Fe II $5316 \AA$ line the intrinsic line profile consists of three components and the situation is more complex. The line width of the components is of about $7 \mathrm{~km} \mathrm{~s}^{-1}$. To roughly estimate the resolution we use the intensity weighted sum of the widths of the components which is of about $12 \mathrm{~km} \mathrm{~s}^{-1}$ and get about 16 elements across the disk or about $12^{\circ}$ in longitude.

\subsection{Doppler imaging with MAXENT}

MAXENT uses MEM to derive the optimum DI solution. A detailed description can be found in Vogt et al. (1987) and in Hatzes et al. (1989). The program maps the line strengths in terms of relative $E W \mathrm{~s}$ on the stellar surface. Free parameters
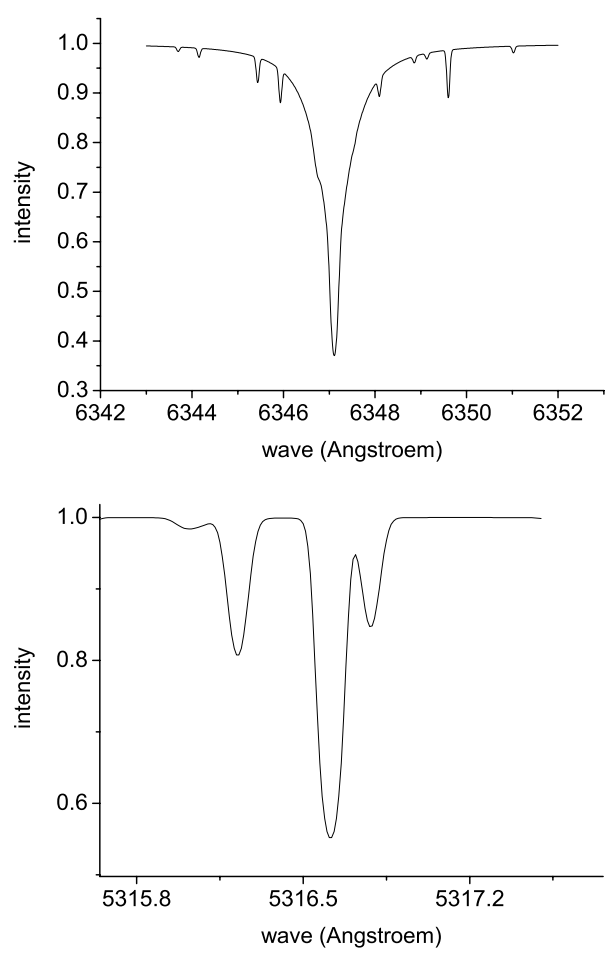

Fig. 1. Intrinsic line profiles calculated with LLmodels and SynthV for $T_{\text {eff }}=14500 \mathrm{~K}, \log g=4.2, \epsilon(\mathrm{Si})=-3.0, \epsilon(\mathrm{Fe})=-3.5$. Top: the Si II $6371 \AA$ line. Bottom: the $5316 \AA$ feature consisting of three (5316.225, 5316.615, and 5316.784 $\AA$ ) Fe II lines.

are the inclination of the rotation axis and the equatorial rotation velocity. In the following, abundance values are given as $\epsilon($ element $)=\log \left(N_{\text {element }} / N_{\text {total }}\right)$.

In the version of the program used, we require intrinsic line profiles that are symmetric about the line centers. This was the reason that we did not apply MAXENT to the iron line (see Fig. 1). Synthetic intrinsic line profiles were generated for each of the silicon lines using LLmodels and SynthV with the model atmosphere parameters $T_{\text {eff }}=14500 \mathrm{~K}, \log g=4.18, \epsilon(\mathrm{Si})=$ $-3.0, \epsilon(\mathrm{Fe})=-3.5$. In the case of the silicon lines the weak blended iron lines were deleted and replaced by a smooth line through the blank space. Then the line was folded about the line center and an average profile, symmetric about the line center was drawn. DI maps were computed for a grid of values of $v \sin i$ and inclination angles $i$. The criterion of the goodness of the fit was the mean rms of the $\mathrm{O}-\mathrm{C}$ values for all 25 profiles. The minimum was found for $v \sin i=100 \pm 3 \mathrm{~km} \mathrm{~s}^{-1}$ and $i=65^{\circ} \pm 5^{\circ}$.

The finally derived $E W$ map for the Si II $6347 \AA$ line is shown in Fig. 2. The local $E W$ scale $\eta$ is in relative units calculated from

$\eta=\log \left(\frac{E W}{\langle E W\rangle}\right)$

where $E W$ is the local equivalent width and $\langle E W\rangle$ is the mean $E W$ over the entire surface. For the Si II lines at 5041, 5056, 6347, and $6371 \AA$ we derived mean $E W$ s of $340,480,620$, and $440 \mathrm{~m} \AA$, respectively. The surface line strength maps derived for the four different silicon lines look very similar and are well presented by Fig. 2 .

The derived map is very detailed, much more detailed than we would expect from our estimations in Sect. 4.1 based on Eq. (2) and the given $v \sin i$ and the FWHM of the intrinsic line profiles (Fig. 1). The program ends up with such a detailed map because it tries to best fit the observed line profiles within a 


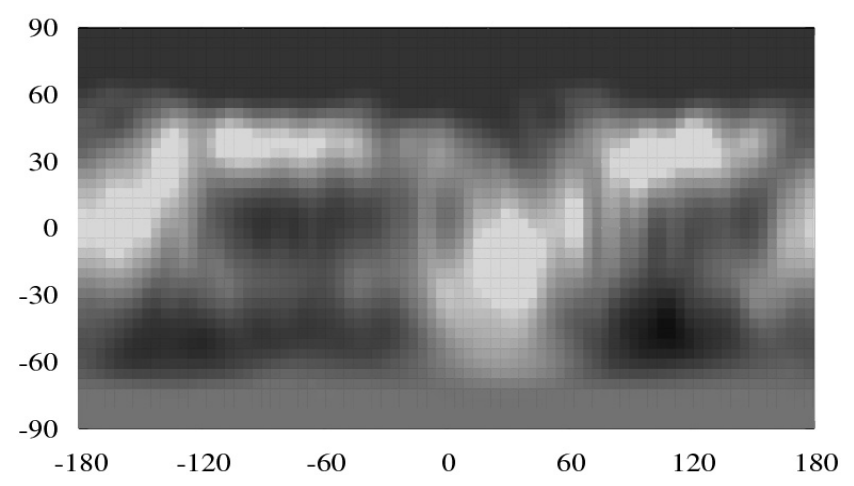

Fig. 2. Surface map (Mercator projection) of the Si II $6347 \AA$ line strengths in terms of relative $E W \mathrm{~s}$ derived with MAXENT. The relative $E W$ scale (see text) spans -0.15 (white) to +0.30 (black).

limit given by the observed signal-to-noise. Besides the alternating regions of high and low line strength in the equator region, MAXENT calculates enhanced line strengths at the pole that directs to the observer. The reason is that the deep cores of the observed line profiles can be fitted only by assuming an elemental concentration at the pole where the variation due to rotation is lowest (see next section).

\subsection{Doppler imaging with INVERS8}

INVERS8 uses a computational algorithm that inverts the timeseries of spectral line profiles into surface maps of chemical abundances. It minimizes the expression

$E(\epsilon)=\sum_{\lambda} \sum_{\varphi}\left(\frac{I_{\lambda \varphi}^{\mathrm{obs}}-I_{\lambda \varphi}^{\mathrm{calc}}(\epsilon)}{\sigma_{\lambda \varphi}}\right)^{2}+\Lambda F(\epsilon)$

where $I_{\lambda \varphi}^{\text {obs }}$ represent the observed spectra and $I_{\lambda \varphi}^{\text {calc }}$ the integrated line profiles calculated from a given surface abundance distribution $\epsilon$. The difference between observed and theoretical spectra is weighted by the individual errors of measurement $\sigma_{\lambda \varphi}$. The summation is carried out over all rotational phases $\varphi$ and wavelengths $\lambda$. INVERS8 uses Tikhonov regularization. $\Lambda$ denotes the regularization parameter and $F$ is the regularization functional calculated from the norm of the abundance gradients, it is

$F(\epsilon)=\sum_{i}\left\|\nabla \epsilon_{i}\right\|^{2}$

index $i$ runs over all surface elements. The optimization algorithm iteratively reduces the total error function $E$ until the discrepancy between the observed and the predicted line profiles is of the same order as the errors of observations (Kochukhov 2003).

INVERS8 uses pre-calculated local intrinsic line profiles, calculated for set of abundances and a given atmosphere model. Input parameters are $T_{\text {eff }}, \log g$, abundance $\epsilon$, equatorial velocity $v$, inclination of the rotation axis $i$, and $\Lambda$. Most parameters were determined in Paper I; $T_{\text {eff }}$ and $\log g$ from the hydrogen line profiles, $v \sin i$ and mean values of the metal abundances from the metal lines. For the determination of the helium abundance we used the BOES spectra that cover a larger wavelength range towards the blue spectral region. We computed a set of atmosphere models with the parameters $T_{\mathrm{eff}}=14000 \mathrm{~K}, 14500 \mathrm{~K}, 15500 \mathrm{~K}$, $\log g=4.2, v \sin i=100 \mathrm{~km} \mathrm{~s}^{-1}$. From these model we calculated the local line profiles for a set of different $\mathrm{Si}$ and $\mathrm{Fe}$ abundances. Using the INVERS8 code we then computed the maps of iron and silicon abundance distributions on the stellar surface. The best solution was found for the model with the parameters $T_{\mathrm{eff}}=$ $14500 \mathrm{~K}, i=65^{\circ}, \epsilon(\mathrm{He})=-3.5, \epsilon(\mathrm{Si})=-3.5, \epsilon(\mathrm{Fe})=-4.0$.

Figure 3 shows $\mathrm{Si}$ and $\mathrm{Fe}$ abundance maps for different values of the regularization parameter $\Lambda$. It should be chosen so that the minimization does not overfit the data, i.e. so that the discrepancy between the observed and the predicted data is of the same order as the errors of measurement. For silicon, the upper, low-resolution map $(\Lambda=0.01)$ is in good agreement with the surface structure that was roughly estimated in Paper I. The stellar surface is dominated by two large, abundance enriched spots on opposite sides and at nearly the same latitude of about $+20^{\circ}$. It is the simplest solution that can explain the basic shape of the observed double-wave in the RV and $E W$ variations of HR 7224. Figure 5 shows the line profiles calculated for $\Lambda=0.01$. It shows that the calculated profiles are not deep enough to fit the cores of the observed profiles. We think that this is due to the low resolution of the resulting DI map. The line cores are filled up only for smaller $\Lambda$ where also structures in the polar region of the star can be resolved.

Our estimation of the possible resolution of about $23^{\circ}$ in longitude for the Si II lines would correspond to a surface map having a resolution more to the middle $(\Lambda=0.001)$ than to the lower $(\Lambda=0.0001)$ panel of Fig. 3. Since the lower panel shows a resolution similar to that obtained with MAXENT we will consider this as the final one, although the results may be slightly overfitted. As in case of the MAXENT results, the line centers are filled up here by the almost non-varying local contributions from the region around the visible pole.

Figure 4 shows the abundance distributions for $\mathrm{Si}$ and $\mathrm{Fe}$ in spherical projection. For silicon, the surface is characterized by two broad regions of lower abundance that divide the equatorial region into four broad regions of alternating lower and higher abundance and give the regions of higher abundance an U-shaped form lying across the upper pole. The abundance is at maximum in the polar region itself. Because of the inclination of the rotation axis of $65^{\circ}$ we can gather no information on the abundance distribution in the region around the other pole.

The derived iron abundance distribution roughly resembles this picture although the obtained structures are not as well defined. The maximum differences in abundance with $1.13 \mathrm{dex}$ for $\mathrm{Si}$ and 1.32 dex for $\mathrm{Fe}$ are comparable.

\subsection{The modified INVERS8 code}

The DI mapping of HR 7224 shows large abundance gradients for all silicon lines and the one iron line used. These gradients change the stellar atmosphere structure in the line formation regions. In Fig. 6 we calculated the temperature in the stellar atmosphere for different $\mathrm{Si}$ and $\mathrm{Fe}$ abundances. Compared to the $\epsilon(\mathrm{Si})=-3.5, \epsilon(\mathrm{Fe})=-3.5$ abundances (solid curve) the temperature increases with increasing silicon abundance (dotted curve) and shows slightly more complex deviations if we change the iron abundance (dashed curve). The change of the stellar atmosphere structure can influence the DI results and we have to consider this influence when we calculate the tables of local line profiles.

As stated above, INVERS8 uses pre-calculated tables of local line profiles, calculated for a set of abundances and a given atmosphere model. We have modified this part of the program by including a multiple-component atmosphere model. In case of HR 7224 we included atmospheres with $T_{\text {eff }}=14500 \mathrm{~K}, \log g=$ 4.2, $\epsilon(\mathrm{Si})=-3.5,-3.0,-2.5$ and $\epsilon(\mathrm{Fe})=-4.0,-3.5,-3.25$. Starting with a single atmosphere model, we calculated a first 

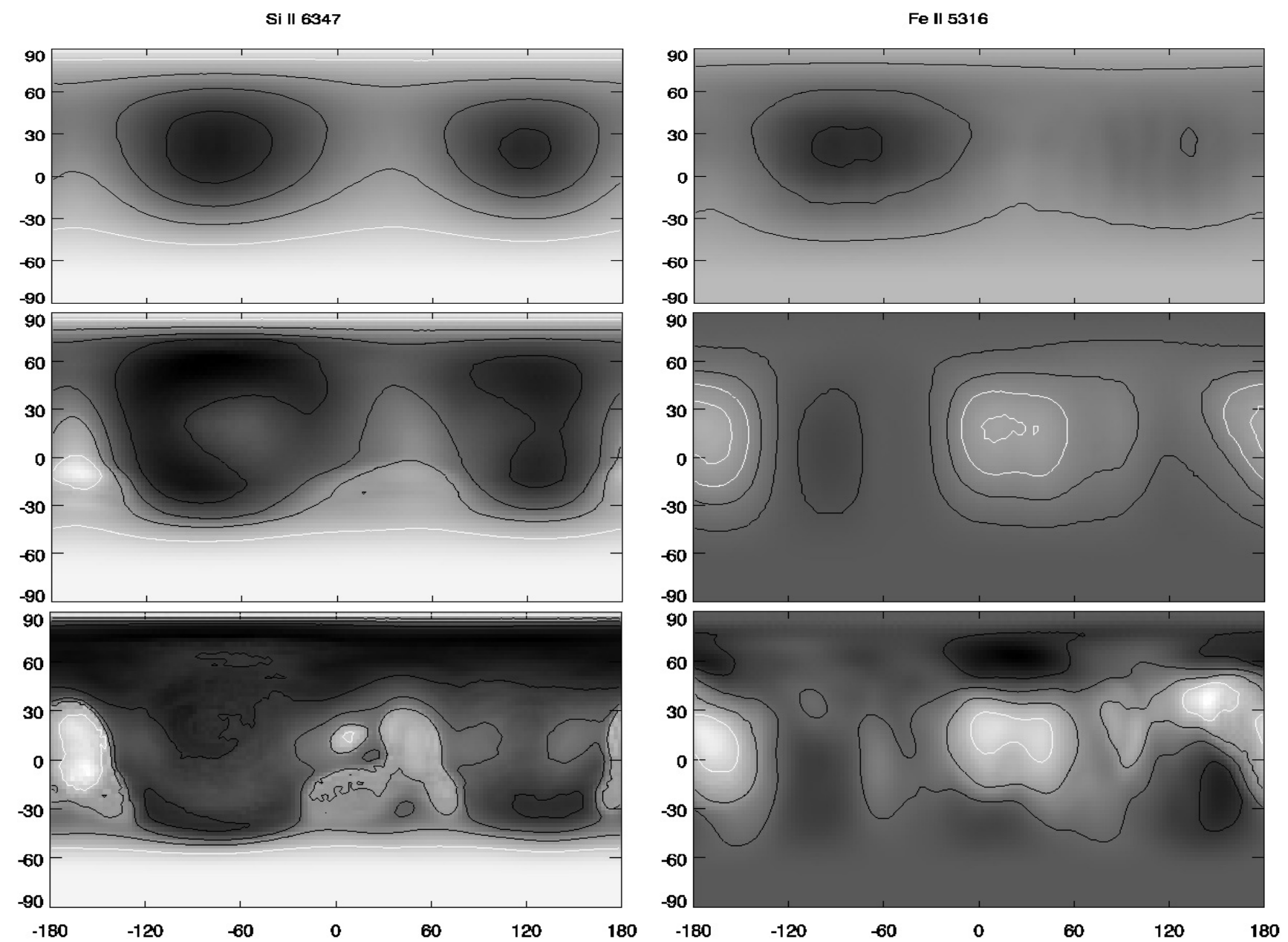

Fig. 3. Stellar surface abundance maps in Mercator projection obtained with INVERS8 from the single atmosphere model with different regularization parameters and $T_{\text {eff }}=14500 \mathrm{~K}, \log g=4.2, \epsilon(\mathrm{Si})=-3.5, \epsilon(\mathrm{Fe})=-4.0, \epsilon(\mathrm{He})=-3.0, v \sin i=100 \mathrm{~km} \mathrm{~s}^{-1}$, $i=65^{\circ}$. From top to bottom: $\Lambda=0.01,0.001,0.0001$. All Si abundances (left) and all Fe abundances (right) are shown on a common dynamical scale reaching from -3.55 (white) to -2.42 (black) for $\mathrm{Si}$ and from -4.36 (white) to -3.04 (black) for Fe.

Si II 6347

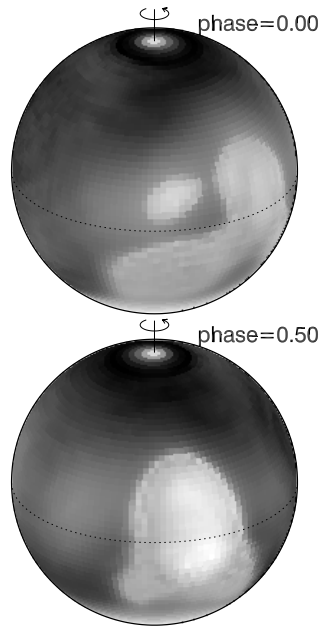

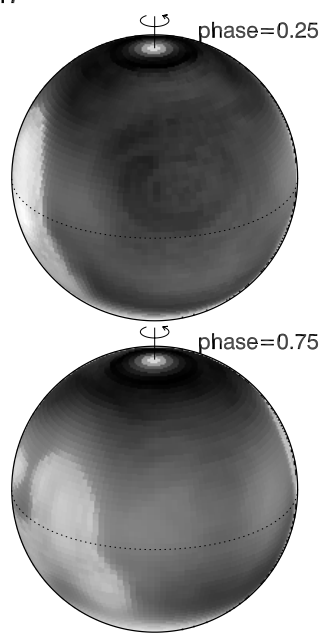
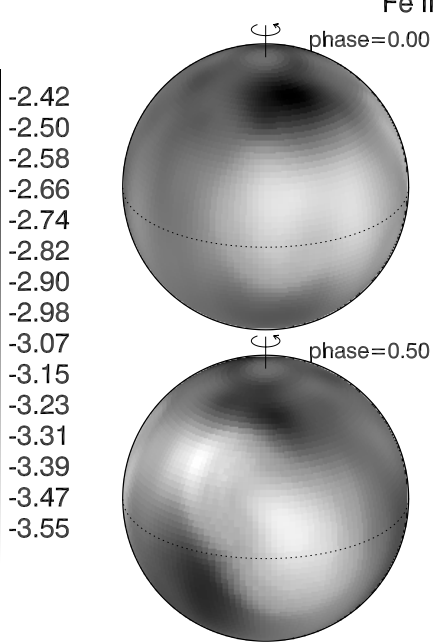

Fe II 5316

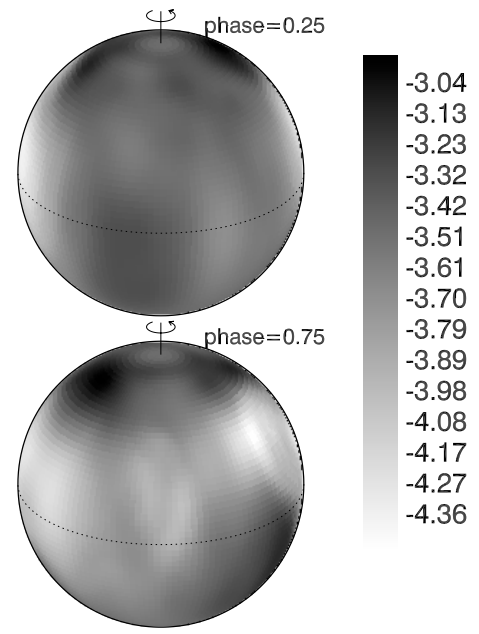

Fig. 4. Stellar surface abundance maps in spherical projection for Si II and Fe II using the same atmospheric parameters as in Fig. 3 and $\Lambda=0.0001$.

abundance map. Then we recalculated the tables of local line profiles by using different atmosphere models for the different abundances obtained from the first DI map. Then the DI procedure was repeated.
The resulting abundance distribution maps can be hardly distinguished by eye from the corresponding single component maps. We therefore only show, in Fig. 7, the differences between the distributions. The difference in silicon abundance is 


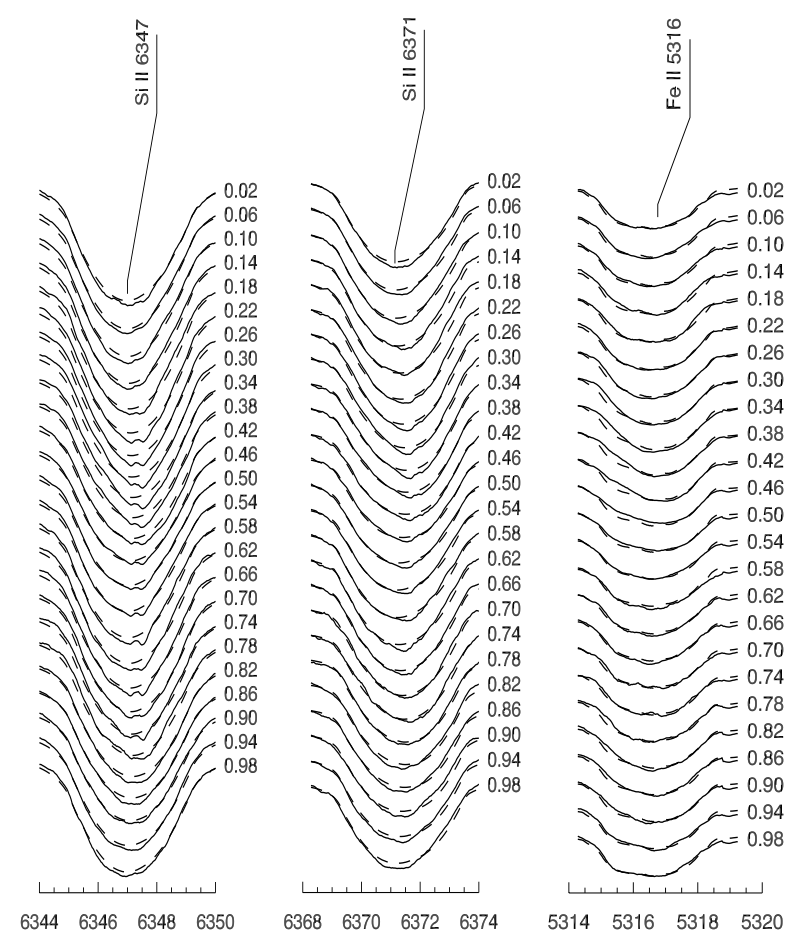

Fig. 5. Comparison between observed (solid) and computed (dashdotted) line profiles derived with INVERS 8 and $\Lambda=0.01$. Central line depths in units of the continuum are $0.81,0.85$, and 0.92 for the Si II 6347 and $6371 \AA$ and the Fe II $5316 \AA$ lines, respectively.

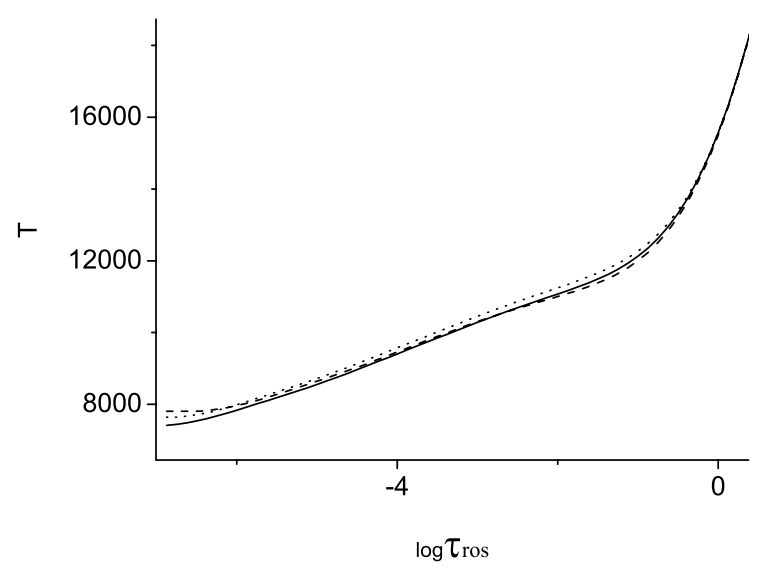

Fig. 6. Change of the stellar structure due to different abundances. Temperature versus Rosseland opacity calculated for $T_{\text {eff }}=14500 \mathrm{~K}$, $\log g=4.2, \epsilon(\mathrm{He})=-3.0$ and $\epsilon(\mathrm{Si})=-3.5, \epsilon(\mathrm{Fe})=-3.5($ solid $) ; \epsilon(\mathrm{Si})=$ $-2.5, \epsilon(\mathrm{Fe})=-3.5($ dotted $)$; and $\epsilon(\mathrm{Si})=-3.5, \epsilon(\mathrm{Fe})=-4.0($ dashed $)$.

very small and of only 0.04 in maximum. The difference in iron abundance reaches a total amplitude of 0.1 . Thus the application of the multiple components atmosphere model does not change in principle the surface abundance distributions obtained with the single components model.

\section{Variability of the hydrogen line profiles}

In Paper I we reported that HR 7224 shows a variability of the hydrogen line profiles during different rotation phases. This variability could not be explained by any spot-like abundance distribution of single elements. It could be shown, however, that the observed variability is in agreement with the assumption of different values of the effective temperature in different rotation

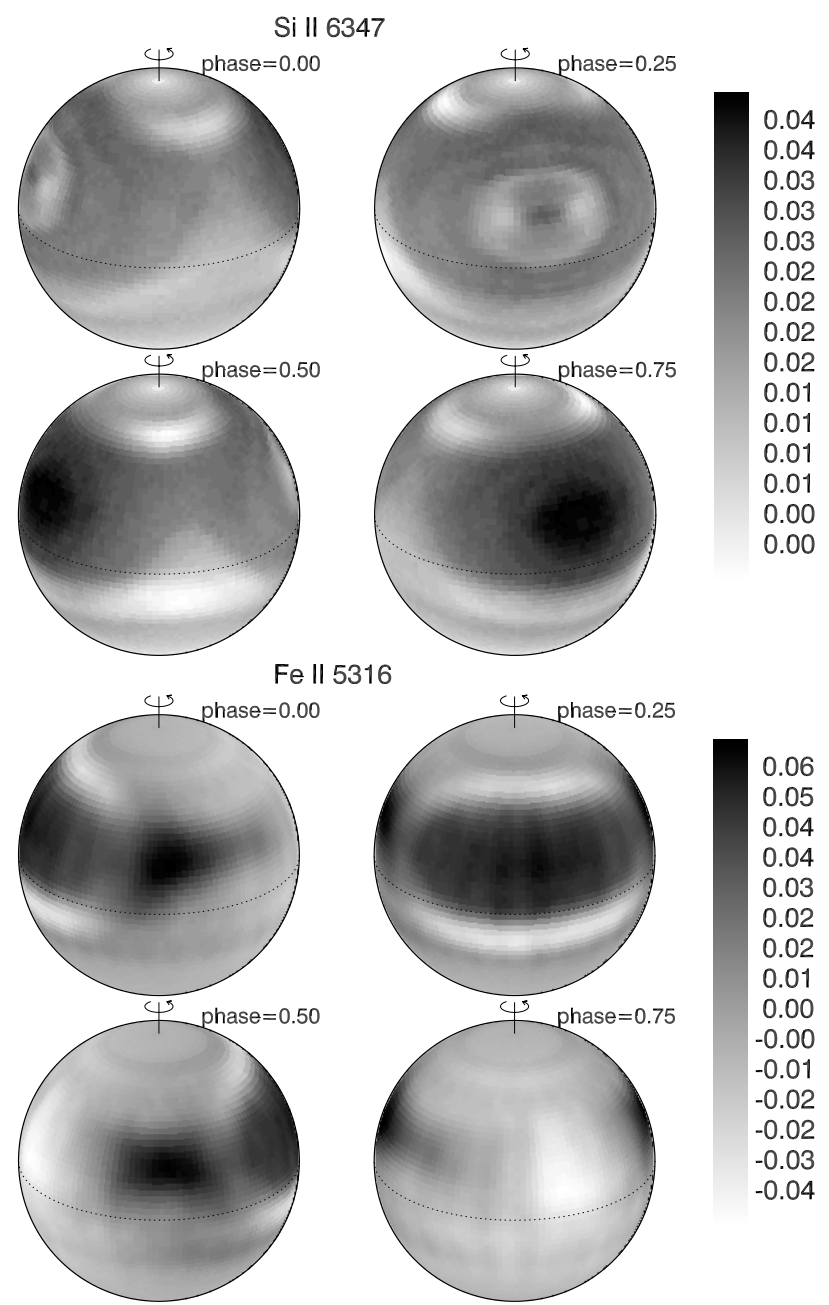

Fig. 7. Maps showing the difference of elemental abundances: multiple minus single components atmosphere model.

phases $\left(T_{\text {eff }}=15500 \mathrm{~K}\right.$ for the phase of minimum and $T_{\text {eff }}=$ $14500 \mathrm{~K}$ for the phase of maximum line strength).

This explanation is non-convincing, in so far as no physical reason for such an inhomogeneous surface temperature distribution is known for this type of star. The most likely reason could be magnetic fields, although in this case we would expect rather a variation of $\log g$ than of $T_{\text {eff }}$ like in case of the Ap stars. As an additional test for the existence of Lorentz forces due to magnetic fields, we computed the standard deviation $\sigma$ of the $\mathrm{H}_{\beta}$ line profiles compared to the mean profile from all rotation phases. If the stellar structure is disturbed due to the presence of non force-free magnetic fields, $\sigma$ should reveal the characteristic fingerprint as the impact of the Lorentz force: the amplitude rises in the wings and drops to the line center (Kroll 1989; Valyavin et al. 2004, 2005; Shulyak et al. 2007). From Fig. 8 we see that HR 7224 does not show the characteristic dip in the line center. Thus we can be relatively sure that HR 7224 shows no changes of $\log g$ from the magnetic pole to the equator caused by Lorentz forces.

In Fig. 9 we compare the observed difference of the $\mathrm{H}_{\beta}$ line profiles between the phases of maximum and minimum line strength with the difference computed from several model atmospheres. Table 1 lists the parameters of the models used. Model I assumes that the temperature is the only parameter that changes. Figure 9 (top) shows that the observed difference in line profiles 


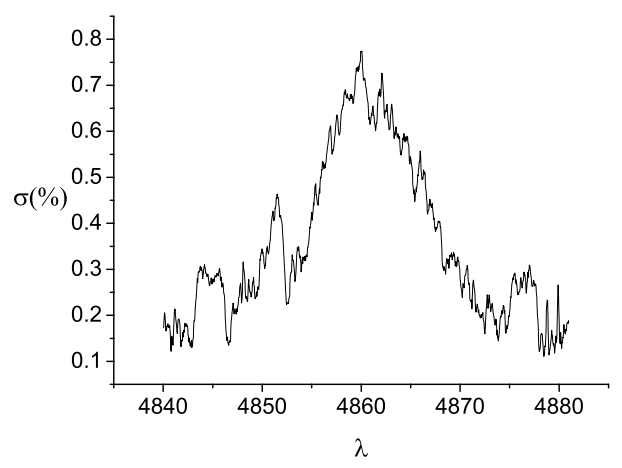

Fig. 8. Standard deviation of the $\mathrm{H}_{\beta}$ lines across the $\mathrm{H}_{\beta}$ profile.

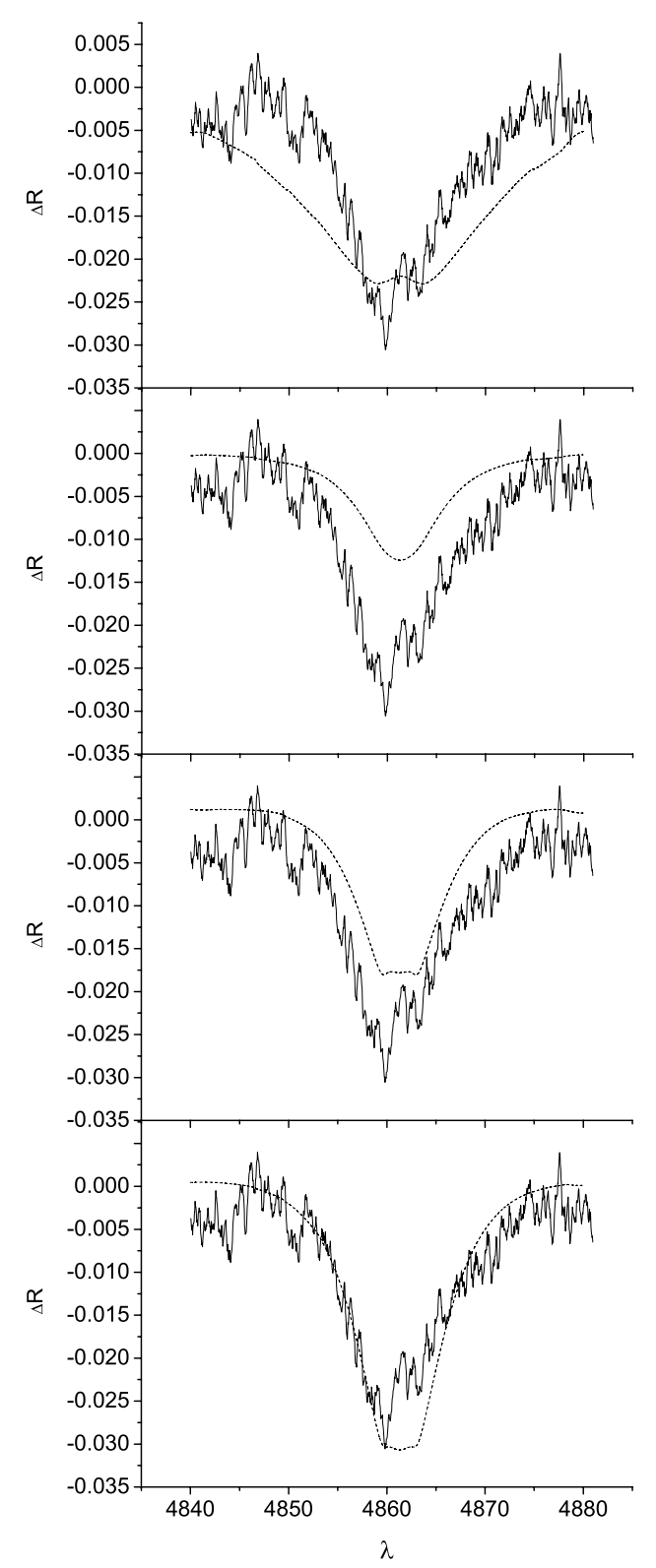

Fig. 9. Solid curves: difference between the $\mathrm{H}_{\beta}$ line profiles at phases of maximum and minimum line strength. Dashed curves: difference computed from synthetic spectra for four different sets of model parameters. From top to bottom: models I, II, III, and IV (see Table 1).

cannot be explained by temperature effects alone. It can work only in combination with elemental abundance changes.
Table 1. Parameters of the four different models for fitting the $\mathrm{H}_{\beta}$ line at phases of minimum and maximum line strength $(\log g=4.2)$.

\begin{tabular}{ccccc}
\hline \hline Model & $T_{\text {eff }}$ & $\epsilon(\mathrm{Si})$ & $\epsilon(\mathrm{Fe})$ & $\epsilon(\mathrm{He})$ \\
\hline I & $\mathbf{1 5 5 0 0}$ & -3.50 & -3.50 & 0.00100 \\
& $\mathbf{1 4 5 0 0}$ & -3.50 & -3.50 & 0.00100 \\
\hline II & 14500 & $\mathbf{- 3 . 5 0}$ & $\mathbf{- 4 . 0 0}$ & 0.00100 \\
& 14500 & $\mathbf{- 2 . 5 0}$ & $\mathbf{- 3 . 2 5}$ & 0.00100 \\
\hline \multirow{2}{*}{ III } & 14500 & -3.50 & -3.50 & $\mathbf{0 . 0 0 1 0 0}$ \\
& 14500 & -3.50 & -3.50 & $\mathbf{0 . 0 7 8 3 4}$ \\
\hline IV & 14500 & $\mathbf{- 3 . 5 0}$ & $\mathbf{- 4 . 0 0}$ & $\mathbf{0 . 0 0 1 0 0}$ \\
& 14500 & $\mathbf{- 2 . 5 0}$ & $\mathbf{- 3 . 2 5}$ & $\mathbf{0 . 0 7 8 3 4}$ \\
\hline
\end{tabular}

In the following, we will try to find out if abundance changes alone can explain the Balmer line variations. In model II to IV we fixed $T_{\text {eff }}$ to $14500 \mathrm{~K}$. Model II assumes that the silicon and iron abundances vary in a maximum range that is compatible to the results of DI mapping. The helium abundance is fixed to the very low value as derived in Paper I. The resulting curve (Fig. 9, second panel) shows the right shape but does not reach the necessary amplitude. This result is in agreement with the results obtained in Paper I.

The helium lines of HR 7224 are very faint and do not allow for a direct investigation of their variations. Despite this faintness, we cannot exclude that there exist helium-enriched spots on the star since they must not give rise to much stronger lines if observed in disk-integrated light. In model III we vary only the helium abundance, between the mean observed value of 0.001 and solar abundance of 0.07834. From Fig. 9, third panel, we can see that the computed variation shows the right shape and reaches almost the necessary amplitude. In our last model IV we vary the abundances of silicon and iron in the maximum range that is compatible with the results if DI mapping and the helium abundance from the observed one to solar abundance. Results show (Fig. 9, bottom panel) that it is possible to fit the observations in this way. The necessary amplitude of the He abundance variation is very large, however.

\section{Discussion}

The observed LPV of the silicon and iron lines has been used to produce surface abundance maps of HR 7224 at different resolutions. In the simplest case, obtained at the lowest resolution, the maps are in good agreement with those expected in Paper I from the double-wave shaped RV and $E W$ variations with rotation. The line cores of the Si II lines are not fitted, however. At higher resolution the line cores are filled up with enhanced contributions from the polar region of the star.

The belts of lower abundance at about $+40^{\circ}$ latitude are present only in the MAXENT map and cannot be found in the INVERS8 map. There are several possible explanations. The relatively large inclination of the rotation axis of the star of $65^{\circ}$ is close to the estimated limit where the north-south ambiguity is too large to give reasonable results (see Sect. 4.1). This could effect the results obtained with MAXENT because the $+40^{\circ}$ latitude where the additional belts occur corresponds to the center latitude of the observed disk. Also an over-fitting of the line profiles or the removing of the weak line blends and the symmetrization of the synthetic intrinsic line profiles (Sect. 4.2) may result in such spurious structures that normally should be washed out according to the estimated possible resolution (Sect. 4.1).

From the DI maps obtained with INVERS8 (Fig. 3) we can see how the surface structure changes from the simplest one - 
only two nearly identical spots on opposite sides of the star, line cores are not fitted - to more detailed structures. Since the rms of the line profile residuals did not change much between $\Lambda=$ 0.001 and $\Lambda=0.0001$ we assume that the optimum case is in between. In both cases the principle surface abundance structure is as described in Sect. 4.3.

The calculated temperature changes in the atmospheric layers due to different abundances of silicon and iron are relatively small (Fig. 6). Nevertheless, we checked for the influence on the computed surface abundance maps by using a multiplecomponent atmosphere model. The differences between the results of the single and the multiple-component atmosphere models are small as well (Fig. 7).

The second panel in Fig. 9 shows that the observed differences in silicon and iron abundances as derived in Paper I give rise to differences in the Balmer line profiles of the right shape compared to the observed ones but not of the sufficient amplitude. The derived metal abundances are precise enough to be sure that the observed Balmer line changes cannot be explained by the abundance changes of silicon and iron alone. Not so for the very weak helium lines that are difficult to measure. This was the reason for us to investigate the additional effect of helium abundance changes. As shown in the bottom panel of Fig. 9, the combined effect of $\mathrm{Si}, \mathrm{Fe}$, and $\mathrm{He}$ abundance changes can explain the observed Balmer line variability. The He abundance gradient has to be very large in this case: He abundance must differ from the observed one up to solar. Such a large gradient is not compatible to the observations since the observed helium line strengths are constant within the errors of measurement in all rotation phases.

The assumption of large temperature gradients on the surface of HR 7224 has the consequence that we cannot interpret the derived DI maps only in terms of abundance distributions, the maps will represent both abundance and temperature variations. This is of interest in particular with respect to the polar region of enhanced silicon line strength: from the curve of growth in the given temperature range we know that enhanced line strength means lower temperature. Thus we possibly observe an analogy to the cool polar caps reported by Böhm-Vitense \& Van Dyk (1987) for the Ap star $\alpha^{2} \mathrm{CVn}$.

There are two main objections against the use of $\alpha^{2} \mathrm{CVn}$ or CU Vir (Kroll 1987) as reference stars for HR 7224, however. First, Böhm-Vitense \& Van Dyk derived for $\alpha^{2} \mathrm{CVn}$ from lightcurve analysis an axial ratio of the star of 0.92 . Such an oblateness due to rotation has the opposite effect, the star should be cooler at the equator than at the poles. Authors cannot give a definite explanation of which mechanism keeps the (magnetic) poles cool. They only speculate that the gas pressure at the poles may be lower due to a pressure stratification by magnetic forces.

And second, from the observational point of view, the findings by Böhm-Vitense \& Van Dyk for $\alpha^{2} \mathrm{CVn}$ and by Kroll (1987), who reported surface temperature variations for the fast rotating Ap star CU Vir, are more or less singular. Kochukhov et al. (2002) investigated the hydrogen line profiles of $\alpha^{2} \mathrm{CVn}$ obtained at different rotation phases and did not find any larger variation of the atmospheric parameters (the shape of the Balmer lines was constant within the noise level of data). And Kuschnig et al. (1999) showed that the variability of the $\mathrm{H}_{\beta}$ line in CU Vir can be explained by surface pressure $(\log g)$ gradients, while $T_{\text {eff }}$ is more or less constant.

Thus the LPV of $\mathrm{H}_{\beta}$ in CUVir is caused by a mechanism that is well known for the Ap stars: strong non force-free magnetic fields disturb the outer layers of the star and produce $\log g$ gradients on its surface. No such mechanism is known for the generation of inhomogeneous surface temperature distributions. And no hint to $\log g$ variations in HR 7224 could be found, neither from the abundance analysis (Paper I) nor from the shape of the standard variation of the $\mathrm{H}_{\beta}$ profiles (Sect. 5).

We could not confirm the existence of stronger magnetic fields of HR 7224 directly by measurement. The two obtained values are very close to each other and indicate a mean longitudinal magnetic field strength in the order of $+400 \mathrm{G}$. The zero field strength is within the $1 \sigma$ limit of errors, however. From the errors of measurement we can estimate an upper limit of $B_{\text {eff }}$ at the date of observation of about $+900 \mathrm{G}$. Considering only the result of the direct magnetic field measurement we cannot exclude the presence of much stronger magnetic fields for two reasons. First, the date of observation corresponds to a rotation phase of 0.53 where the strength of silicon lines is close to minimum (see Fig. 10 in Paper I). This rotation phase could correspond to the cross-over phase of the magnetic field. And second, without any knowledge on the timely variations of the magnetic field observed during rotation we cannot exclude magnetic field configurations where the local magnetic field strength is much stronger than observed in the mean longitudinal case. Our check for the presence of Lorentz forces by means of the standard deviation of the $\mathrm{H}_{\beta}$ line profiles, on the other hand, makes it unlikely that stronger magnetic fields on HR 7224 exist.

\section{Conclusions}

The use of two different methods of Doppler Imaging, MAXENT (MEM) and INVERS8 (Tikhonov regularization), gave comparable results for the basic structure of the silicon abundance distribution. In both final maps the equatorial region is divided into four large regions of alternating lower and higher abundance (line strength). And in both maps the upper pole that directs towards the observer shows enhanced silicon abundance. Neglecting the existence of temperature variations, the observed abundance gradients of $\mathrm{Si}$ and Fe are large, larger than 1 dex for both elements.

We could show that it is not possible to explain the observed variability of the Balmer lines by surface abundance gradients alone or by pressure or $\log g$ gradients. At present state, the LPV of Balmer and metal lines can be explained only if we also assume that surface temperature gradients exist. The order of the assumed amplitude of the temperature variation of $1000 \mathrm{~K}$ is also in agreement with the amplitude of the observed photometric variations as we could show in Paper I. In consequence the obtained DI maps represent also a temperature distribution and the star is cooler at the visible pole.

The question if HR 7224 possesses stronger magnetic fields remains open. The results of our attempt to measure the field strength do not exclude such a possibility, but further magnetic field measurements that cover the full rotation cycle of HR 7224 are urgently needed.

At present state the physical reason for the existence of the assumed surface temperature gradients remains unknown.

Acknowledgements. Authors would like to thank A. Hatzes for his assistance in applying the MAXENT program code, N. Piskunov for providing us with the INVERS 8 code, D. Shulyak for the calculation of the atmosphere models, and G. Valyavin for providing the software for magnetic field measurements. L.F. acknowledges the support of his work by the Brazilian agency $\mathrm{CNPq}$ through the grants Nos. 290086/2004-3 and 154566/2006-3. V.T. is thankful to Austrian Science Fund (FWF) P17890. D.M. acknowledges his work as part of the research activity of the Astrophysical Center for the Structure and Evolution of the 
Cosmos (ARCSEC) which is supported by the Korean Science and Engineering Foundation.

\section{References}

Adelman, S. J. 1997, A\&AS, 122, 249

Adelman, S. J. 2004, MNRAS, 351, 823

Bohlender, D. A., Landstreet, J. D., \& Thompson, I. B. 1993, A\&A, 269, 355

Böhm-Vitense, E., \& Van Dyk, S. D. 1987, AJ, 93,1527

Cohen, J. G. 1970, ApJ, 159, 473

Cowley, A., Cowley, Ch., Jaschek, M., et al. 1969, AJ, 74, 375

Crawford, D. L. 1963, ApJ, 137, 530

Farnsworth, G. 1932, ApJ, 76, 313

Hatzes, A. P., Penrod, G. D., \& Vogt, S. S. 1989, ApJ, 341, 456

Kim, K. M., Jang, B. H., Han, I., et al. 2002, J. Korean Astron. Soc., 35, 221

Kim, K. M., Han, I., Valyavin, G. G., et al. 2007, PASP, in press

Kochukhov, O. 2003, Ph.D. Thesis, Uppsala University

Kochukhov, O., Piskunov, N. E., Ilyin, I., et al. 2002, A\&A, 389, 420
Kroll, R. 1989, Rev. Mod. Astron., 2, 194

Kuschnig, R., Ryabchikova, T. A., Piskunov, N. E., et al. 1999, A\&A, 348, 924 Landstreet, J. D. 1980, AJ, 85, 661

Lehmann, H., Tsymbal, V., Mkrtichian, D. E., et al. 2006, A\&A, 457, 1033 (Paper I)

Monin, D. N., Fabrika, S. N., \& Valyavin, G. G. 2002, A\&A, 396, 131

Piskunov, N. E., \& Rice, J. B. 1993, PASP, 105, 1415

Piskunov, N. E., \& Kochukhov, O. 2002, A\&A, 381, 736

Piskunov, N. E., Kupka, F., Ryabchikova, T. A., et al. 1995, A\&AS, 112, 525

Shulyak, D., Tsymbal, V., Ryabchikova, T., et al. 2004, A\&A, 428, 993

Shulyak, D., Valyavin, G., Kochukhov, O., et al. 2007, A\&A, 464, 1089

Tsymbal, V. 1996, ASP Conf. Ser., 108, 198

Valyavin, G., Kochukhov, O., Piskunov, N., et al. 2004, A\&A, 420, 993

Valyavin, G., Kochukhov, O., Shulyak, D., et al. 2005, J. Korean Astron. Soc., 38,283

Vogt, S. S., Penrod, G. D., \& Hatzes, A. P. 1987, ApJ, 321, 496

Wade, G. A., Donati, J.-F., Landstreet, J. D., et al. 2000, MNRAS, 313, 851

Winzer, J. E. 1974, Ph.D. Thesis, Univ. Toronto 